\title{
UN MÉTODO DE BAJO COSTO PARA LA DETERMINACIÓN DE COBRE A NIVEL DE VESTIGIOS EN MATRICES DE INTERÉS AMBIENTAL POR ESPECTROFOTOMETRÍA EN FASE SÓLIDA (EFS)
}

\author{
Roberto Gerardo Pellerano, Cesar Hamilton Romero, Hugo Arnoldo Acevedo y Francisco Antonio Vazquez* \\ Facultad de Ciencias Exactas Naturales y Agrimensura, Universidad Nacional del Nordeste, Av. Libertad 5.450, \\ Corrientes (3400), Argentina
}

Recebido em 29/9/06; aceito em 27/4/07; publicado na web em 25/10/07

\begin{abstract}
A LOW-COST PROCEDURE FOR COPPER DETERMINATION IN NATURAL WATERS AND PLANT MATERIALS EXPLOITING SOLID PHASE SPECTROPHOTOMETRY. A method for determining copper by solid phase spectrophotometry (SPS) was optimized using the Doehlert design. Copper(II) was sorbed on a styrene-divinylbenzene anion-exchange resin as a $\mathrm{Cu}$ (II)-1-(2-pyridylazo)-2-naphthol (PAN) complex, at pH 7.0. Resin phase absorbances at 560 and $800 \mathrm{~nm}$ were measured directly. The detection limit was found to be $2.5 \mu \mathrm{g} \mathrm{L}^{-1}$. The relative standard deviation on ten replicate determinations of $10 \mu \mathrm{g} \mathrm{Cu}(\mathrm{II})$ in $1000 \mathrm{~mL}$ samples was $1.1 \%$. The linear range of the determination was 5.0-100 $\mu \mathrm{g} \mathrm{L}{ }^{-1}$. The method was applied successfully to the determination of $\mathrm{Cu}(\mathrm{II})$ in natural water and vegetable samples.
\end{abstract}

Keywords: copper; solid phase spectrophotometry; natural water.

\section{INTRODUCCIÓN}

La presencia del cobre en el ambiente (agua, aire y suelo), esta ligada principalmente a la meteorización de las formaciones rocosas y a la actividad volcánica que son fuentes de una concentración basal, en las aguas naturales por ejemplo. Habiendo sido referidas para las mismas concentraciones comprendidas en el rango 1-20 $\mu \mathrm{g} \mathrm{L}{ }^{-1}$, para aguas dulces superficiales, y del orden de $0,15 \mu \mathrm{g} \mathrm{L}^{-1}$, para aguas marinas en Argentina ${ }^{1}$. La cantidad del elemento es incrementada por aportes antropogénicos, los que incluyen emisiones de la actividad minera, de las fundiciones que lo utilizan y de otras industrias. $\mathrm{El} \mathrm{Cu}$ es asimismo incorporado a algunos procesos de tratamientos de aguas para el control de algas.

La deficiencia de este elemento en la nutrición humana puede provocar anomalías como la anemia o mal formaciones óseas, por lo que el $\mathrm{Cu}$ se considera como elemento esencial ${ }^{2}$. Vestigios de $\mathrm{Cu}$ intervienen en reacciones metabólicas formando parte de diversas proteínas ${ }^{3}$, tales como la ceruloplasmina, la citocromoxidasa, etc. Sin embargo la exposición crónica a altas concentraciones de $\mathrm{Cu}(\mathrm{II})$ produce efectos tóxicos ${ }^{4}$. Esto determina interés en el monitoreo de $\mathrm{Cu}(\mathrm{II})$ a nivel de vestigios en muestras ambientales.

$\mathrm{Si}$ bien existen numerosas técnicas para determinar $\mathrm{Cu}(\mathrm{II})$ a nivel de vestigios estas son de carácter instrumental (absorción atómica ${ }^{5}$, espetroscopía de emisión atómica por plasma ${ }^{6}$, técnicas electroquímicas ${ }^{7}$, etc.) que presentan como principal desventaja el elevado costo de instalación y mantenimiento. La espectrofotometría UV-vis en solución para la determinación de $\mathrm{Cu}(\mathrm{II})$, si bien resulta económica, incorpora pasos previos a la determinación, generalmente para aumentar la sensibilidad, provocando mayores riesgos de contaminación y aumento del tiempo necesario para cada determinación.

En este trabajo se propone la espetrofotometría en fase sólida ${ }^{8}$ (EFS) como vía alternativa para la medición de concentraciones de $\mathrm{Cu}(\mathrm{II})$ a nivel de vestigios. Esta metodología presenta como ventajas la sencillez, sensibilidad, versatilidad y facilidad de aplicación además del bajo requerimiento de instrumental, dado que se lleva a cabo mediante el uso de un espectrofotómetro UV-vis convencional.

*e-mail: fvazquez@exa.unne.edu.ar
Con el objeto de obtener las condiciones óptimas para el método se utilizó el modelo de superficie de respuesta basado en el diseño de Doehlert ${ }^{9}$.

\section{PARTE EXPERIMENTAL}

\section{Instrumental}

Espectrofotómetro UV-visible haz simple (200 - $1100 \mathrm{~nm})$, marca Metrolab ${ }^{\circledR} 1700$, controlado desde PC, utilizando el programa Metrolab ${ }^{\circledR} \mathrm{SF} 170^{10}$. Cubetas de cuarzo (Hellma ${ }^{\circledR} 100$ QS) de 1 $\mathrm{mm}$ de paso óptico y volumen útil de $0,240 \mathrm{~mL}$, con adaptador para portacubetas de fabricación propia.

$\mathrm{pH}$-metro digital, electrodo combinado de vidrio, marca Altronix ${ }^{\circledR}$ TPX. Centrifuga marca Cavour $^{\circledR}$ VT-32165-DC 0 - 3500 rpm. Agitador mecánico rotativo.

\section{Reactivos}

Solución reguladora $0,1 \mathrm{~mol} \mathrm{~L}-1$ de fosfato a base $\mathrm{KH}_{2} \mathrm{PO}_{4}$ y de $\mathrm{NaOH}$, con pH ajustado a 7,0; solución de tensioactivo no iónico éter mono[p-(1,1,3,3, tetrametilbutil) fenílico] del polietilenglicol (Triton X-100, Merck ${ }^{\circledR}$ ) 5\% (v/v); solución Stock: concentración de $\mathrm{Cu}$ (II) $500 \mathrm{mg} \mathrm{L}^{-1}$; solución etanólica de 1-(2-pyridylazo)-naftol (PAN) de concentración $2 \times 10^{-3} \mathrm{~mol} \mathrm{~L}^{-1}$; resina Dowex 1 X-8 200 400 mallas. La resina se utilizó en forma clorulada.

Todos los reactivos fueron preparados con agua de elevada pureza (bidestilada sobre vidrio).

\section{Software}

Unscrambler V 6.011 para el diseño experimental y Origin 7.0 [9] para gráficos.

\section{Determinación de $\mathrm{Cu}(\mathrm{II})$ por EFS}

La EFS está basada en la preconcentración de un analito, formando un compuesto coloreado (Cu(II)-PAN), sobre un sustrato sólido (resina de intercambio iónico Dowex 1 X-8) y medición de la absorbancia del 
compuesto coloreado directamente sobre la fase sólida. Este proceso permite obtener mejor sensibilidad y precisión que los métodos de absorción en solución. La reacción del ion $\mathrm{Cu}$ (II) con PAN se lleva a cabo en solución acuosa, pero dada la insolubilidad del reactivo PAN y sus complejos, en agua, se procedió al agregado de un tensoactivo; el elegido fue Tritón X100 (tensoactivo no iónico). Luego se fijó el complejo coloreado sobre una resina aniónica tipo Dowex 1 X-8.

\section{Medidas de absorbancia en EFS}

En EFS la absorbancia de un determinado compuesto fijado sobre una resina se mide en forma diferente a la espectrofotometría UV-vis en solución.

En este trabajo la absorbancia del producto de la reacción fijado en la resina se midió a $560 \mathrm{~nm}$ (pico de máxima absorción para el complejo Cd (II) - PAN), y a $800 \mathrm{~nm}$ (esta última longitud de onda corresponde a la zona donde solo la resina causa atenuación de la luz por empaquetamiento). Luego por diferencia entre ambas absorbancias encontradas previamente se obtiene la absorbancia neta proporcional a la concentración de compuesto fijado. Similarmente las absorbancias de los blancos correspondientes fueron medidas a las mismas longitudes de onda.

La absorbancia neta, $A_{\text {neta }}$, para el compuesto se calcula:

$A_{\text {neta }}=A_{\text {complejo }}-A_{B C O}$

donde: $A_{\text {complejo }}=A_{560}-A_{800}$ para la muestra y $A_{B C O}=A_{560}-A_{800}$ para el blanco.

\section{Diseño experimental}

Entre los diferentes diseños de superficie de respuesta posibles se eligió el diseño de Doehlert dado que ofrece una distribución uniforme de puntos en el espacio de respuesta experimental. Dicha uniformidad de la distribución describe una figura romboidal (en el caso de dos variables se produce un hexágono), en la que se asignan cinco niveles a la variable de mayor peso o importancia (PAN, en este caso) y tres a la otra variable (Tritón X-100). Requiere también un escaso número de determinaciones experimentales, generados a partir de $N=k^{2}+k+1$. Esto representa la principal ventaja de este diseño con respecto a otros métodos de amplia aplicación, como el diseño central compuesto, por ejemplo, provocando en consecuencia un importante ahorro de costo-tiempo.

Se obtienen entonces siete puntos experimentales que deben medirse, para poder procesar y obtener los coeficientes de regresión cuadrática de la siguiente ecuación:

$A_{\text {neta }}=a+b[P A R]+C[$ Triton $]+d[P A N]^{2}+e[\text { Triton }]^{2}+f[P A N][$ Triton $]$

Donde: $a$ es el término independiente, $b$ y $c$ son coeficientes de los términos lineales, $d$ y $e$ son coeficientes cuadráticos y $f$ es el coeficiente de interacción. Cada coeficiente representa la proporción de la variabilidad total de la variable dependiente (absorbancia neta, ABS), que es explicada por la ecuación de regresión.

\section{RESULTADOS Y DISCUSIÓN}

\section{Parámetros que afectan la determinación de $\mathrm{Cu}$ (II) por EFS}

En la EFS el primer objetivo que se plantea es la elección del soporte adecuado sobre el que se fijará el compuesto en estudio. Se realizaron, en placa de toque, varios ensayos cualitativos de fijación del compuesto en los soportes aniónicos: Dowex 1 X-8 y de adsorción: Amberlite XAD 7 y Amberlite XAD 16, realizando simultáneamente ensayos en blanco para cada uno.

Se eligió el soporte aniónico Dowex 1 X-8, que produce fijación rápida y completa. Con el resto de las resinas la fijación no fue completa, esto se pone en evidencia porque la solución permanece coloreada luego de un tiempo de contacto prudencial y además, presentan la desventaja de una elevada opacidad al paso de la luz a las longitudes de onda de trabajo.

Otro parámetro importante porque afecta en forma directa la sensibilidad del método es el volumen de trabajo, en esta ocasión se fijó un volumen de $50 \mathrm{~mL}$ de muestra.

\section{Dependencia del pH}

La influencia del $\mathrm{pH}$ sobre la formación del complejo $\mathrm{Cu}$ (II)PAN se estudió en solución, dado que la formación del complejo $\mathrm{Cu}$ (II)-PAN se realizó en la fase líquida previo a su fijación en la fase sólida (resina). Se toman $100 \mathrm{~mL}$ de muestra conteniendo al menos $0,1 \mathrm{mg}$ de $\mathrm{Cu}(\mathrm{II})$, y se le adicionaron a continuación los siguientes reactivos: $0,5 \mathrm{~mL}$ Tritón X-100 (5\% v/v) y $0,5 \mathrm{~mL}$ de solución de PAN $1 \times 10^{-3} \mathrm{~mol} \mathrm{~L}^{-1}$. Finalmente se hizo variar el $\mathrm{pH}$ de la solución obtenida agregando soluciones de $\mathrm{HCl} 0,1 \mathrm{~mol} \mathrm{~L}^{-1} \mathrm{e} \mathrm{NaOH}$ $0,1 \mathrm{~mol} \mathrm{~L}^{-1}$. Los valores de $\mathrm{pH}$ ensayados estuvieron comprendidos entre 4,5 y 10,5. La gráfica obtenida de representar los diferentes valores de absorbancia neta medidos versus los diferentes valores de pH medidos potenciométricamente, luego de homogeneizar cuidadosamente las soluciones, puede verse en la Figura 1.

\section{Gráfico de Pareto estandarizado para Abs}

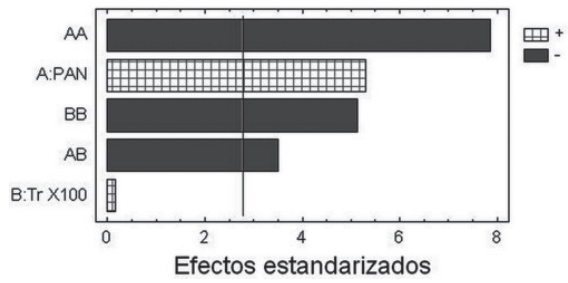

Figura 1. Influencia del pH de la formación del complejo Cu(II)-PAN. Absorbancia medida a $555 \mathrm{~nm}$ en solución

Se observaron valores prácticamente constantes en el rango de 6,2 a 7,4. El valor de pH óptimo seleccionado fue de 7.0 el cual fue ajustado utilizando solución reguladora de $0,1 \mathrm{~mol} \mathrm{~L}^{-1}$ de fosfato a base $\mathrm{KH}_{2} \mathrm{PO}_{4}$ y de $\mathrm{NaOH}$ por no ocacionar interferencias perceptibles en este rango de aplicación.

\section{Diseño para optimizar [PAN] y [Tr X-100]}

El primer diseño propuesto se encuentra centrado en el punto $[\mathrm{PAN}]=6 \times 10^{-6} \mathrm{~mol} \mathrm{~L}^{-1} \mathrm{y}[\operatorname{Tr} \mathrm{X}-100]=0,05 \%$, dicho punto se replica tres veces para obtener una estimación del error experimental. Las coordenadas del modelo y las absorbancias medidas se pueden observar en la Tabla 1.

Para la optimización se utilizaron alícuotas de $50 \mathrm{~mL}$ de solución tipo de $\mathrm{Cu}(\mathrm{II})$ de concentración adecuada, luego se agregaron sucesivamente volúmenes variables de acuerdo con el punto experimental, solución de Tritón X-100 + solución etanólica de PAN + $1 \mathrm{~mL}$ de buffer $\mathrm{pH}$ 7,0 + $70 \mathrm{mg}$ de resina aniónica Dowex 1 X-8.

A continuación se agita la muestra en agitador mecánico durante $20 \mathrm{~min}$. Se separa la resina del seno de la solución luego de centrifugar 5 min a $1.500 \mathrm{rpm}$. Se decanta por succión de la solución sobrenadante con vacío. Se cargan con la ayuda de goteros de vidrio las cubetas de $1 \mathrm{~mm}$ de paso óptico. Finalmente se lee la absorbancia 
en el espectrofotómetro siguiendo el procedimiento explicado anteriormente para la medición de absorbancia en EFS.

Luego del análisis de regresión se obtiene una tabla de análisis de la varianza (ANOVA) correspondiente al modelo, Tabla 2 y una gráfica de superficie de respuesta Figura 2.

Tabla 1. Diseño para optimizar [PAN] y [Tr X-100].

Se pueden observar los niveles de concentración tenidos en cuenta para la regresión de superficie de respuesta. Los valores han sido distribuidos teniendo en cuenta el modelo de Doehlert

\begin{tabular}{lccc}
\hline Experimento & $\begin{array}{c}\text { PAN } \\
{\left[\mathrm{x} 10^{-6} \mathrm{~mol} \mathrm{~L}^{-1}\right]}\end{array}$ & $\begin{array}{c}\text { Tr X-100 } \\
{\left[\mathrm{x} 10^{-2} \%\right]}\end{array}$ & $\begin{array}{c}\text { Absorbancia } \\
\text { Neta }\end{array}$ \\
\hline 1 & $6(-1)$ & $5(0)$ & 0.220 \\
2 & $8(-0.5)$ & $7.5(+1)$ & 0.218 \\
3 & $8(-0.5)$ & $2.5(-1)$ & 0.278 \\
4 & $10(0)$ & $5(0)$ & 0.226 \\
5 & $12(+0.5)$ & $7.5(+1)$ & 0.292 \\
6 & $12(+0.5)$ & $2.5(-1)$ & 0.223 \\
7 & $14(+1)$ & $5(0)$ & 0.308 \\
\hline
\end{tabular}

Tabla 2. Tabla de análisis de la varianza correspondiente al modelo de regresión aplicado

\begin{tabular}{lccccc}
\hline Fuente & $\begin{array}{c}\text { Suma de } \\
\text { cuadrados }\end{array}$ & $\begin{array}{c}\text { Grados de } \\
\text { libertad }\end{array}$ & $\begin{array}{c}\text { Cuadrado } \\
\text { medio }\end{array}$ & F & P \\
\hline A: PAN & 0,013441 & 1 & 0,0134 & 28,08 & 0,006 \\
B: Tr X-100 & 0,000011 & 1 & 0,0000 & 0,02 & 0,886 \\
AA & 0,029502 & 1 & 0,0295 & 61,63 & 0,001 \\
AB & 0,005852 & 1 & 0,0059 & 12,22 & 0,025 \\
BB & 0,012567 & 1 & 0,0126 & 26,25 & 0,007 \\
Error Total & 0,001915 & 4 & 0,0005 & & \\
Total & 0,089302 & 9 & & & \\
\hline
\end{tabular}

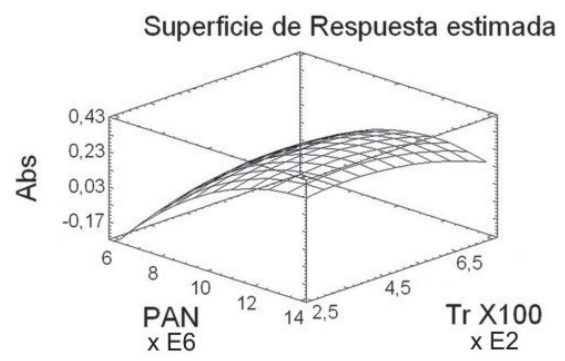

Figura 2. Superficie de respuesta obtenida para el diseño 1 con aplicación del modelo de Doehlert

La tabla de ANOVA divide la variabilidad de la repuesta (Abs) en distintos segmentos separados para cada uno de las variables y sus interacciones, después prueba la significación estadística de cada efecto comparando el cuadrado de la media contra una estimación del error experimental. En este caso, 4 de los efectos tienen los p-valores inferiores a 0,05 , indicando que son significativamente diferentes de cero al $95,0 \%$ de nivel de confianza. Esto puede observarse también utilizando el gráfico de Pareto, que es un gráfico de barras que permite observar la significación de cada variable y sus interacciones sobre la variable de respuesta, Figura 3. El estadístico R-cuadrado indica que el modelo así ajustado explica el 97,9\% de la variabilidad. El estadístico R-cuadrado ajustado, el cual es más adecuado para la comparación de números diferentes de variables independientes, es 95,17\%. El error estándar de la estimación muestra la desviación normal de los residuos es 0,0219. El estadístico Durbin-Watson (DW) examina los residuos para determinar si hay cualquier correlación significativa basada en el orden en el que se suceden en el fichero de datos. Puesto que el p-valor es superior a 0,05 , no hay indicios de correlación de serie en los residuos, lo asegura la validez del modelo aplicado.

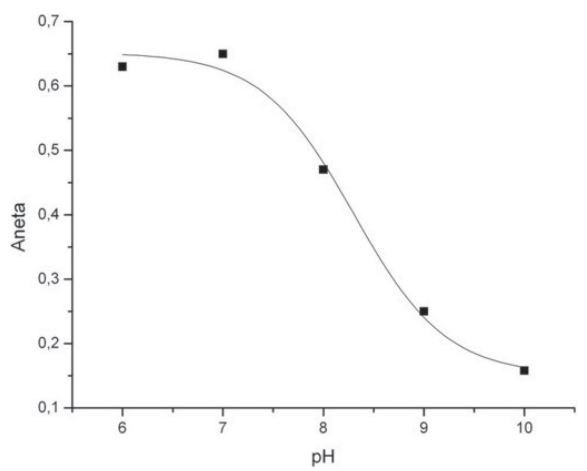

Figura 3. Gráfico de Pareto que permite observar el peso de cada variable sobre la variable de respuesta y sus interacciones

La superficie de respuesta, según la aplicación del criterio de Lagrange ${ }^{11}$, indica que aparece un máximo de respuesta en las siguientes coordenadas para una absorbancia neta máxima igual a 0,329 Coordenadas: $[\mathrm{PAN}]=13,25 \times 10^{-6} \mathrm{~mol} \mathrm{~L}^{-1}$, $[\operatorname{Tr} \mathrm{X}-100]=3,99 \times 10^{-2} \%$, que como se puede observar, el máximo aparece en la zona lateral derecha de la superficie definida por el campo experimental, Figura 2. Teniendo en cuenta que el máximo se encuentra en una zona de meseta de la superficie de respuesta se realiza una elección de compromiso final para las condiciones optimas para la reacción con el $\mathrm{Cu}$ (II).

$[P A N]=1,3 \times 10^{-5} \mathrm{~mol} \mathrm{~L}^{-1} \mathrm{y}[\operatorname{Tr} \mathrm{X}-100]=4,0 \times 10^{-2} \%$

\section{Otros parámetros experimentales}

Se estudió el tiempo de agitación necesario para lograr la fijación completa del complejo a la resina, y se encontró como óptimo en 20 min. El complejo fijado permanece estable sobre la resina al menos por $48 \mathrm{~h}$ después de la fijación. La secuencia de adición de reactivos determinada es la siguiente: $50 \mathrm{~mL}$ de muestra + Triton X-100 + PAN + solución reguladora + resina aniónica.

La masa de resina es otro parámetro a tener en cuenta ${ }^{12}$, se fijó el valor en $70 \mathrm{mg}$ de resina, que es una masa suficiente para que una vez cargada en la cubeta alcance a cubrir el camino óptico.

\section{Propiedades analíticas}

Se realizó una curva de calibración bajo las condiciones óptimas encontradas. La gráfica de calibración resulta lineal entre 5 y $100 \mu \mathrm{g} \mathrm{L}^{-1}$ de $\mathrm{Cu}(\mathrm{II})$, la pendiente alcanzó un valor de $0,012{\mathrm{~L} \mu \mathrm{g}^{-1}}$ con un coeficiente de correlación de $0,995(\mathrm{n}=6)$. Se calculó el límite de detección en $2,5 \mu \mathrm{g} \mathrm{L}^{-1}$ teniendo en cuenta el criterio de $3 \sigma$ o tres veces la desviación estándar de una serie de 10 determinaciones de la absorbancia neta correspondiente al blanco ${ }^{13}$. La reproducibilidad se calculó realizando determinaciones independientes conteniendo $10 \mu \mathrm{g} \mathrm{L}^{-1}$ de $\mathrm{Cu}(\mathrm{II})$, obteniéndose un valor de $1,7 \%$.

El límite de detección obtenido por el método propuesto se encuentra en niveles similares a otras técnicas instrumentales, tales como las de espectrometría atómica ${ }^{14-16}$ o espectrometría atómica combinadas con espectrometría de masas ${ }^{17}$, aunque los costos de instalación-mantenimiento no son comparables. Los valores de los 
límites de detección típicos de las diferentes técnicas instrumentales disponibles pueden observarse en la Tabla 3.

El límite de detección de la técnica propuesta en este trabajo (EFS) es coherente con las demás técnicas instrumentales sofisticadas. La principal desventaja de la EFS es el elevado tiempo requerido por cada determinación.

\section{Efecto de iones extraños}

Se realizó un estudio de los posibles interferentes para la determinación de $\mathrm{Cu}$ (II). Para ello se prepararon soluciones tipo que contenían $10 \mathrm{mg} \mathrm{L}^{-1}$ de $\mathrm{Cu}(\mathrm{II})$, los valores de concentración informados de los interferentes son tales que el error relativo introducido en la determinación fue inferior a $\pm 5 \%$.

Los resultados se presentan en la Tabla 4. Se observan interferencias importantes, principalmente producidas por cationes tales como $\mathrm{Fe}(\mathrm{III}), \mathrm{Zn}$ (II), Ni(II) y $\mathrm{Co}(\mathrm{II})$. Se estudió el agregado de citrato de sodio como agente enmascarante, a una concentración de 1 x $10^{-3} \mathrm{~mol} \mathrm{~L}^{-1}$ que resultó eficiente para eliminar la interferencia debida a los iones $\mathrm{Zn}$ (II), Fe(III), Cd(II) y $\mathrm{Pb}(\mathrm{II})$. La interferencia debida al $\mathrm{Co}(\mathrm{II})$ y $\mathrm{Ni}(\mathrm{II})$ no resulta de importancia a niveles normales de aparición en aguas naturales de río.

Tabla 3. Comparación del límite de detección del método propuesto con respecto a otras técnicas instrumentales de elevado costo

\begin{tabular}{llc}
\hline Técnica & & $\begin{array}{c}\text { Límite de } \\
\text { detección } \\
{\left[\mu \mathrm{g} \mathrm{L}^{-1}\right]}\end{array}$ \\
\hline $\begin{array}{l}\text { Espectrometría de } \\
\text { absorción atómica }\end{array}$ & Llama (FAAS) ${ }^{20}$ & $1,0-3,0$ \\
$\begin{array}{l}\text { Espectrometría de } \\
\text { emisión atómica }\end{array}$ & $\begin{array}{l}\text { Horno de grafito (GFAAS) })^{16} \\
\text { inductivo (ICP-AES) }\end{array}$ & 1,0 \\
& $\begin{array}{l}\text { Plasma de acoplamiento } \\
\text { inductivo - Espetrometría } \\
\text { de masa (ICP-MS) }\end{array}$ & 2,0 \\
Espectrometría en & Método propuesto & 0,8 \\
Fase Sólida (EFS) & & 2,5 \\
\hline
\end{tabular}

Refs.16-19
Tabla 4. Estudio del efecto de posibles interferentes en la determinación de $10 \mu \mathrm{g} \mathrm{L^{-1 }}$

\begin{tabular}{lc}
\hline Ion extraño & $\begin{array}{c}\text { Concentración tolerada con } \\
\text { enmascarante }\left[\mu \mathrm{L} \mathrm{L}^{-1}\right]\end{array}$ \\
\hline $\mathrm{Cl}^{-}, \mathrm{NO}_{3}^{-}, \mathrm{NO}_{2}^{-}, \mathrm{SiO}_{3}^{2-}, \mathrm{PO}_{4}^{3-}$, & 10000 \\
$\mathrm{CO}_{3}^{2-}, \mathrm{HCO}_{3} \mathrm{Ca}(\mathrm{II}), \mathrm{Mg}(\mathrm{II})$ & 5000 \\
$\mathrm{SO}_{4}^{2-}$ & 2000 \\
$\mathrm{~F}^{-}$ & 1000 \\
$\mathrm{Al}(\mathrm{III})$ & 100 \\
$\mathrm{Cr}(\mathrm{III}), \mathrm{Pb}(\mathrm{II}), \mathrm{Fe}(\mathrm{III})$ & 50 \\
$\mathrm{Cd}(\mathrm{II})$ & 25 \\
$\mathrm{Bi}(\mathrm{III}), \mathrm{Zn}(\mathrm{II})$ & 10 \\
$\mathrm{Ni}(\mathrm{II})$ & 5 \\
$\mathrm{Co}(\mathrm{II})$ &
\end{tabular}

\section{Aplicaciones analíticas}

El método de EFS propuesto se aplicó a diferentes matrices de interés ambiental, como: agua de río, agua potabilizada y muestras de origen vegetal como hojas de té verde y hojas de yerba mate. Los resultados se presentan en la Tabla 5.

Para validar los valores de concentración obtenidos se determinó en forma paralela el contenido de $\mathrm{Cu}$ (II) con espectrofotometría de absorción atómica con horno de grafito ${ }^{18}$ (GFAAS), se observó gran concordancia de los valores de concentración medidos por las técnicas en las diferentes muestras.

Finalmente como una aplicación particular, el contenido de $\mathrm{Cu}$ (II) en el agua potabilizada de la ciudad de Corrientes se encontró por debajo del límite de detección propuesto. Se realizó el estudio de sobreagregado de estándar ${ }^{19}$ a la muestra para comprobar el desempeño del método desarrollado. Se obtuvieron excelentes porcentajes de recuperación en todos los casos (superior al 95\%) Tabla 5.

\section{CONCLUSION}

Se consiguió desarrollar un método para la determinación de $\mathrm{Cu}(\mathrm{II})$, sencillo confiable, sensible y de bajo costo aplicable a muestras con matrices de interés ambiental.

Tabla 5. Aplicaciones analíticas - Concentraciones de $\mathrm{Cu}(\mathrm{II})$ medidas con el método propuesto y con la técnica de referencia GFAAS

\begin{tabular}{|c|c|c|c|c|}
\hline Muestra & $\mathrm{Cu}(\mathrm{II})$ sobreagregado $\left[\mu \mathrm{g} \mathrm{g}^{-1}\right]$ & $\mathrm{Cu}(\mathrm{II})$ encontrado $^{\mathrm{a}}\left[\mu \mathrm{g} \mathrm{g}^{-1}\right]$ & GF-AAS $\left[\mu \mathrm{g} \mathrm{g}^{-1}\right]$ & Recuperación [\%] \\
\hline Hojas de té verde & - & $26,4 \pm 0,4$ & $26,9 \pm 0,1$ & 98,1 \\
\hline Hoja de té negro & - & $22,2 \pm 0,5$ & $21,8 \pm 0,2$ & \\
\hline \multirow[t]{2}{*}{ Hojas de Yerba Mate } & - & $47,2 \pm 0,4$ & $46,9 \pm 0,1$ & 100,6 \\
\hline & & {$\left[\mu \mathrm{g} \mathrm{L}^{-1}\right]$} & {$\left[\mu \mathrm{g} \mathrm{L}^{-1}\right]$} & \\
\hline Agua Río Paraná & - & $24,5 \pm 0,4$ & $25,0 \pm 0,1$ & 98,0 \\
\hline \multirow[t]{4}{*}{ Agua de bebida } & - & $<2,5$ & $<1,0$ & - \\
\hline & 10 & 10,4 & - & 104 \\
\hline & 30 & 30,5 & - & 101,6 \\
\hline & 50 & 50,1 & - & 100,2 \\
\hline
\end{tabular}

\footnotetext{
${ }^{a}$ Promedio de tres determinaciones
}

\section{REFERENCIAS}

1. Subsecretaría de recursos hídricos de la Nación; Desarrollos de Niveles Guia Nacionales de Calidad de Agua Ambiente Correspondientes a Cobre, Niveles Guía Nacionales de Calidad de Agua Ambiente, 2005

2. Nath, R.; Int. J. Biochem. Cell Biol 1997, 29,1245

3. Aitio, A.; Aro, A.; Jarvisalo, J.; Vainio, H.; Trace elements in health and disease, $1^{\text {st }}$ ed., RSC: Cambridge, 1991
4. Malvankar, P. L.; Shinde, V. M.; Analyst 1991, 116, 1081.

5. Welz, B.; Atomic Absorption Spectroscopy, $1^{\text {st }}$ ed., Wiley: Amsterdam, 1985, cap. 9.

6. Kamiura, T.: Funasaka, K.; Tajima, Y.; Kawaraya T.; Kuroda, K.; Anal. Chim. Acta 1996, 327, 61.

7. Chow, C. W. K.; Kolev, S. D.; Davey D. E.; Mulcahy, D. E.; Anal. Chim. Acta 1996, 330, 79.

8. Yoshimura, K.; Waki, H.; Talanta 1985, 32, 345. 
9. Doehlert, D. H.; Applied Statistics 1970, 19, 231

10. Metrolab SF-170; Software for spectrophotometer, Metrolab, Argentina, 1999.

11. The Unscrambler 6.11, CAMO® Computer Aid Modelling, 1996

12. Zougagh, M.; Rudner, P. C.; De Torres, A. G.; Pavon, J. M. C.; J. Anal. At. Spectrom. 2000, 15, 1589

13. Ortega-Barrales, P.; Fernández de Córdova; M. L.; Molina-Díaz, A.; Anal. Chim. Acta 1998, 376, 227

14. IUPAC; Pure Appl. Chem. 1976, 45, 115

15. U.S. EPA National Exposure Research Laboratory (NERL); Method 200.7 Determination of Metals and Trace Elements in Water and Wastes by Inductively Coupled Plasma-Atomic Emission Spectrometry, 1994.
16. U.S. EPA National Exposure Research Laboratory (NERL); Method 200.9. Determination of Trace Elements by Stabilized Temperature Graphite Furnace Atomic Absorption, 1994.

17. U.S. EPA National Exposure Research Laboratory (NERL); Method 200.8 Determination of Trace Elements in Waters by Inductively Coupled Plasma - Mass Spectrometry, 1994.

18. Cabon, J. Y.; Le Bihan, A.; Spectrochim. Acta, Part B 1995, 50, 1703.

19. Prichard, E.; Mackay, G. M.; Points, J.; Trace Analysis: A structures approach to obtaining reliable results, $1^{\text {st }}$ ed., RSC: United Kingdom, 1996. 